\title{
Determination of cannabinoids in Cannabis sativa L. samples for recreational, medical, and forensic purposes by reversed-phase liquid chromatography-ultraviolet detection
}

\author{
Sanja Zivovinovic ${ }^{\dagger}$, Ruth Alder $^{\dagger}$, Martina D. Allenspach and Christian Steuer ${ }^{*}$ (D)
}

\begin{abstract}
Background: Currently, an increasing demand of cannabis-derived products for recreational and medical use is observed. Therefore, the reliable and fast quantification of cannabinoids in hemp samples is essential for the control of product from Cannabis sativa, L. strains. In general, gas chromatography (GC) is the method of choice for the quantification of cannabinoids whereas this method is time consuming and the detection of acidic precursor is not feasible without derivatization.

Methods: We report the successful development and validation of an accurate and broadly applicable reversedphase high-performance liquid chromatography (RP-HPLC) method coupled to an ultra violet (UV) detector including an optimized extraction procedure for the separation and quantification of eight different cannabinoids.

Results: The optimized method is able to separate cannabidivarin, cannabidiolic acid, cannabigerolic acid, cannabigerol, cannabidiol, cannabinol, $\Delta$ 9-tetrahydrocannabinol, and tetrahydrocannabinolic acid within $10 \mathrm{~min}$. For all target analytes, the \%-Bias at the lower and upper calibration range varied from -1.3 to $10.3 \%$ and from -3.9 to $8.6 \%$, respectively. The most suitable agent for extracting cannabis plant samples was evaluated to be a mixture of acetonitrile and water in a ratio 1:1. The extraction efficiency was more than 95\% for all analytes in recreational hemp samples. Stability studies on acidic cannabinoids showed a high likeliness of decarboxylation at $100^{\circ} \mathrm{C}$ and aromatization after exposure to UV light, respectively. A modified loss on drying method revealed a moisture content between 4 and 10\%. The developed method was successfully applied to measure the cannabinoid content in recreational and forensic hemp samples representing broad range of cannabinoid amounts and patterns.

Conclusion: The present work proposes validated methods for the determination of cannabinoids in cannabis samples. The use of RP-HPLC-UV renders this method broadly applicable and allows the detection of acidic precursor in even less time compared to GC-based methods.
\end{abstract}

Keywords: HPLC, Method development, Validation, Cannabinoids

\footnotetext{
* Correspondence: christian.steuer@pharma.ethz.ch

+Sanja Zivovinovic and Ruth Alder contributed equally to this work.

Department of Chemistry and Applied Biosciences, Institute of

Pharmaceutical Sciences, Swiss Federal Institute of Technology (ETH),

Vladimir-Prelog-Weg 4, 8093 Zürich, Switzerland
} 


\section{Introduction}

Since centuries, Cannabis sativa L. (C. sativa) is used for industrial purposes but it is better known as illegal drug possessing psychotropic properties. However, C. sativa is also a highly decorated medicinal plant for the use as anticancer agent, for neuroprotection and as bone marrow stimulants (Velasco et al. 2016; Machado Rocha et al. 2008). With the legalization of cannabis for therapeutic use, the demand for pure and characterized samples has grown significantly (Corroon and Phillips 2018). Therefore, currently new pharmacopeial monographs are in development for quality control of C. sativa-based medicinal products (Pavlovic et al. 2018). Besides the medical use, there is an enormous interest from consumers/patients in the utilization of low $\Delta 9$-tetrahydrocannabinol (THC) hemp for recreational use. In recent years, a kind of gold-rush fever is observed in Europe and all over the world and many new suppliers entered the market (Pellechia 2018). Since there is a complicated and different legislation for $C$. sativa products all over Europe, caution for quality control has to be taken. Although there is no upper limit for the cannabidiol (CBD) or cannabidiolic acid (CBDA) content in most European countries, maximum limits of THCor $\Delta 9$-tetrahydrocannabinolic acid (THCA) contents vary between 0.1 and $1 \%$ within Europe.

Cannabinoids belong to terpenophenolic compounds and are the main constituents of the cannabis plant. Terpenoids and phenols were also identified in the cannabis plant but are of lower pharmacological importance (Pavlovic et al. 2018). Cannabigerolic acid (CBGA) is the starting point in the biosynthetic pathway of all cannabinoids, which are synthesized in vivo in a carboxylated form (Fig. 1). In the plant, CBDA and THCA are synthesized by enzymatic catalyzed reactions. However, ex vivo stress conditions like heat and UV light decompose these precursors to their decarboxylated form: CBGA $\rightarrow$ cannabigerol (CBG), $\mathrm{CBDA} \rightarrow \mathrm{CBD}$ and THCA $\rightarrow$ THC, respectively (Citti et al. 2018a; Sirikantaramas and Taura 2017). Under UV light, $\triangle 9$-THC is further aromatized to cannabinol (CBN). THC and $\mathrm{CBD}$ are two main biomarkers in commercial available hemp samples. THC is mostly responsible for psychotropic activities whereas CBD is more anxiolytic and sleep inducing. In comparison to THC, CBD is not considered a controlled substance. Numerous reports have been published for the qualitative and quantitative analysis of cannabinoids in cannabis and its preparations. This study will therefore focus on those substances for possible therapeutic use such as CBD, $\triangle 9$-THC, CBG, CBN, cannabidivarin (CBDV), cannabichromene, and tetrahydrocannabivarin (Amada et al. 2013; Thomas et al. 2007). Several comprehensive reviews of the chemical analysis of cannabis plants, corresponding preparations, and forensic specimens were presented in the past (Citti et al. 2018b; Wang et al. 2017; Patel et al. 2017; ElSohly and Salem 2000). The most widespread techniques applied for separation were gas chromatography (GC) with and without derivatization,<smiles>C=C(C)C1CCC(C)=CC1c1c(O)cc(CCC)cc1O</smiles> 
high-performance liquid chromatography (HPLC), and to a lesser extend supercritical fluid chromatography (Wang et al. 2016; U.N.O.o. Drugs, Crime 2013). The GC method is still officially employed by the authorities for the determination of cannabinoids. But it is obvious that acidic forms are not accessible without prior derivatization, and further conversion of THCA to THC is not quantitative at all (Dussy et al. 2005). Some researchers postulate that an accurate cannabinoid profile should be evaluated by determining the acid and neutral forms separately (Pavlovic et al. 2018; Citti et al. 2018b; Calvi et al. 2018; Ambach et al. 2014). On the other hand, LC-based procedures render the derivatization step superfluous and enable the detection of the heat-labile acid precursor in less time. However, determining chromatographic conditions is more challenging. Additionally, the pre-analytical phase has to be taken into account for method development and validation. Extraction, storage conditions, and stability determination play a pivotal role in the analysis of $C$. sativa L.-derived products (Dussy et al. 2005; Brighenti et al. 2017; Mudge et al. 2017).

The main scope of this study was the development and validation of a fast and convenient UV-detector-based RP-HPLC method for the fast quantification of cannabinoids in CBD samples and forensic cannabis samples. The present study examines further pre-analytical conditions and the analytical stability of cannabinoids under different stress conditions. Eight authentic CBD-hemp materials and 12 forensic cannabis samples offering a wide range of cannabinoid patterns were analyzed. Results of the overall THC-content of forensic samples were compared with gas chromatographic method (U.N.O.o. Drugs, Crime 2013), the formerly gold standard in cannabinoid analysis. Additionally, a modified loss on drying method was applied to determine the moisture content of all cannabis samples. Finally, the developed method was transferred easily to an ultra-high performance liquid chromatography (UHPLC) device using know metrics, thus further reducing analysis time from 10 to less than $5 \mathrm{~min}$.

\section{Materials and methods}

Analytical standards were obtained from Lipomed (Reinach, Switzerlanf). Formic acid (FA), methanol (MeOH), ethanol $(\mathrm{EtOH})$, and acetonitrile $(\mathrm{ACN})$ were obtained from Merck (Darmstadt, Germany) and were of LCMS grade. Pure-water was generated from an in-house water purification system from Labtec (Villmergen, Switzerland). For all experiments, Gilson DIAMOND tips were used. Hop strobiles (Humulus lupulus L.) were obtained from local pharmacies. CBD-hemp tobacco samples were purchased from several licensed producers within Switzerland. The Forensic Institute Zurich (Zurich, Switzerland) provided 12 forensic cannabis samples.

\section{Chromatographic analysis HPLC conditions}

Reversed-phase chromatography was done using a LaChrom Elite System (Hitachi, Ltd., Tokio, Japan) HPLC system consisting of a LaChrom Elite L-2200 autosampler, a LaChrom Elite L-2130 pump, a LaChrom Elite L-2350 column oven, and a LaChrom Elite L-2420 UV-VIS detector. For peak integration, Agilent EZChrom Elite was used. The final liquid chromatography analysis was performed on a Phenomenex Kinetex XB-C18 column $(150 \times 4.6 \mathrm{~mm}, 2.6 \mu \mathrm{m})$ applying gradient elution, using pure-water (with $0.1 \% \mathrm{FA}$ ) and acetonitrile (with $0.1 \% \mathrm{FA}$ ) as the organic phase. The injection volume was $15 \mu \mathrm{L}$, and the dwell volume of the HPLC system was $1.8 \mathrm{~mL}$. The column-oven temperature was set to $50^{\circ} \mathrm{C}$, and the flow rate was $0.8 \mathrm{~mL} / \mathrm{min}$. Monitoring of all cannabinoids was done at $\lambda=220 \mathrm{~nm}$.

\section{UHPLC conditions}

Reversed-phase chromatography was done using a HITACHI ChromasterUltra UHPLC system consisting of a 6270 autosampler, a 6310 column oven, a 6170 binary pump, and a 6430 Diode Array Detector. For peak integration, Agilent EZChrom Elite was used. The final liquid chromatography analysis was performed on a Phenomenex Kinetex XB-C18 column $(150 \times 2.1 \mathrm{~mm}$, $1.7 \mu \mathrm{m}$ ) applying gradient elution, pure-water (with $0.1 \%$ formic acid), and acetonitrile (with $0.1 \%$ formic acid) as the organic phase. The injection volume was $5 \mu \mathrm{L}$, and the dwell volume of the UHPLC system was $0.7 \mathrm{~mL}$. The column-oven temperature was set to $50^{\circ} \mathrm{C}$, and the flow rate was $0.8 \mathrm{~mL} / \mathrm{min}$. Monitoring of all cannabinoids was done at $\lambda=220 \mathrm{~nm}$.

\section{Extraction}

All preliminary extraction experiments were performed using sample (A). Twenty milligrams of sample was extracted with $2.5 \mathrm{~mL}$ solvent in a cooled ultrasonic bath. Afterwards, samples were centrifuged at $10^{\circ} \mathrm{C}$ for $15 \mathrm{~min}$ at $4000 \mathrm{rpm}$. Supernatant was filtered using a PFTE filter $(0.45 \mu \mathrm{m}$, Machery Nagel $)$ prior to analysis and tenfold diluted with solvent. Recovery effect (RE) was tested at QC low level using three independent spiked hop samples.

\%Recovery effect $(\mathrm{RE})=\left(\frac{\text { Response }_{\text {extracted sample with analytes }}}{\text { Response }_{\text {post-extracted spiked sample }}}-1\right) \times 100$

Extraction efficiency (EE) was determined in triplicate extracting CBD and THC rich samples three times.

\section{Validation}

Commercially available $1 \mathrm{mg} / \mathrm{mL}$ methanolic solutions of all analytes were used as stock solutions for calibration and QC spiking solutions. Four different concentrations 


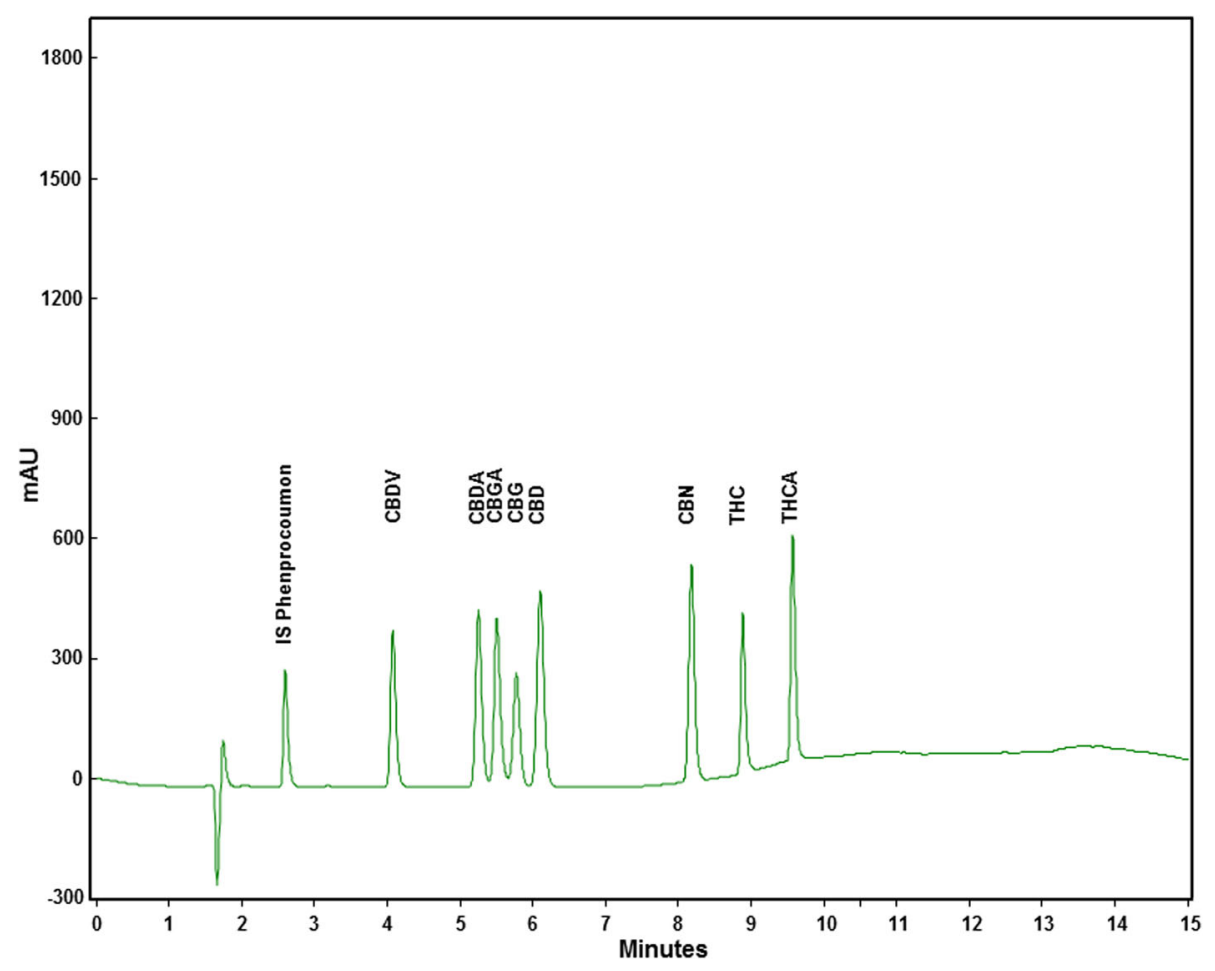

Fig. 2 UV chromatogram of Cal 2 recorded at $\lambda=220 \mathrm{~nm}$

of the analytes in the range of $1-100 \mu \mathrm{g} / \mathrm{mL}$ were chosen. Working solutions were prepared by serial dilution from each stock solution in methanol. QC low and QC high samples were analyzed in duplicate on each of 6 days. Accuracy was given in terms of bias as the percent deviation of the mean calculated concentration compared to the theoretical value. Intra-day and inter-day imprecision was calculated as relative standard deviation (RSD) according to Peters et al. (2009). Phenprocoumon was used as internal standard (IS) at a final concentration of $200 \mu \mathrm{g} / \mathrm{mL}$.

\section{Stability studies}

Stability of cannabinoids was tested in an oven (VD20 Binder, Huber) at $100{ }^{\circ} \mathrm{C}$ and under UV light (Honle, Sol 2, 350-700 nm). For the heat stability experiment, the sample was placed in weighing flasks. For the UV stability, one weighing flask was covered with aluminum foil and the other was exposed to UV light. For both stability experiments, $20 \mathrm{mg}$ of sample was taken from each of the flasks at indicated time points and was analyzed. At indicated time points, $20 \mathrm{mg}$ of sample was taken from each of the vials and analyzed. All stability experiments were performed in duplicate.

\section{Quantification}

Authentic samples were extracted and quantified applying the developed and validated method. All samples $(20 \mathrm{mg})$ were analyzed in duplicate. The final amount of analyte [\%] was calculated using the dilution factor given by the procedure and the weighed amount of plant sample. The determined concentration of the authentic forensic samples was compared to those obtained by established GC-FID-based method as described previously (U.N.O.o. Drugs, Crime 2013). (Details can be found in Additional file 1).

\section{Loss on drying}

Loss on drying experiment of hop was performed in an oven (VD20 Binder, Huber) $105^{\circ} \mathrm{C}$ for $2 \mathrm{~h}$ (Pharmacopoea europaea (Ph. Eur.) 2.2.32) (Ph. Eur., Loss on Drying (2.2.32) 2018). Cannabis samples were placed in weighing flasks and were dried to constant mass at $60^{\circ} \mathrm{C}$.

\section{Results and discussion}

\section{Chromatographic analysis}

Reversed phase chromatography RP-HPLC was chosen for the separation of eight cannabinoids. The focus was set on C18 columns, since these were the most commonly used in the literature. Several C18 columns with different eluent compositions, flow rates, and column temperatures were tested (Additional file 1: Table S1). A baseline separation of all analytes was finally achieved using the Kinetex XB-C18 HPLC column $(2.6 \mu \mathrm{m}, 150 \times$ $4.6 \mathrm{~mm}$,) with $\mathrm{H}_{2} \mathrm{O} / 0.1 \%$ FA and ACN/0.1\% FA as solvent. Flow rate and temperature was set to $0.8 \mathrm{~mL} / \mathrm{min}$ 
and $50{ }^{\circ} \mathrm{C}$, respectively (Additional file 1 : Table S2). Selected cannabinoids were separated within $10 \mathrm{~min}$ under HPLC conditions (Fig. 2). After cleaning and reequilibration, complete run time of this method was $20 \mathrm{~min}$. The resolution of all analytes was at least $R_{\mathrm{s}}>1.7$ and therefore in the acceptable range for quantification. The asymmetry factor of all peaks is between 1.2 and 1.5 (Table 1). Although for peaks 3 and 4, resolution and asymmetry factor were not in the optimal range, validation data in terms of bias and imprecision for CBGA(3) and CBG(4) were acceptable. The same column was used by Mudge et al. (2017)and De Backer et al. (2009) (Citti et al. 2018b) to separate the same number of cannabinoids, but with separation times of $14 \mathrm{~min}$ and $20 \mathrm{~min}$, respectively. As internal standard (IS), phenprocoumon was used. Under selected chromatographic conditions, a clear separation between the IS and all cannabinoids was achieved. Finally, the developed HPLC method was transferred to an UHPLC system coupled to a diode array detector (DAD). The chemistry of the column (Kinetex C18, $1.7 \mu \mathrm{m}, 150 \times$ $2.1 \mathrm{~mm}$ ) was similar to the HPLC column, and the same mobile phases were used. The injection volume was reduced to $5 \mu \mathrm{L}$. Target analytes were separated in less than 5 min (Additional file 1: Figure S5). The resolution of all peaks was above 1.5 and the asymmetry (10\%) between 0.9 and 1.1.

\section{Extraction}

The most commonly used extracting agent for cannabinoids according to literature (Patel et al. 2017; U.N.O.o. Drugs, Crime 2013; De Backer et al. 2009; Zoller et al. $2000)$ is a mixture of methanol $(\mathrm{MeOH})$ and chloroform $\left(\mathrm{CHCl}_{3}\right)$ in a ratio of 9:1. Considering the volatility and toxicity of chloroform and taking green chemistry guidelines into consideration, this halogenated solvent should not be the solvent of choice. Performance of other extracting solvents and solvent mixtures were compared to the standard $\mathrm{MeOH} / \mathrm{CHCl}_{3}$ (9:1) mix. One of these mixtures was $\mathrm{MeOH} / \mathrm{H}_{2} \mathrm{O}$ (4:1) which is also used by Mudge and coworkers (Mudge et al. 2017). The other

Table 1 Retention times and chromatographic values of each analyte obtained by the HPLC method

\begin{tabular}{llll}
\hline$R_{\mathrm{t}}(\mathrm{min})$ & Analyte & Resolution $R$ & Asymmetry (10\%) \\
\hline 4.2 & 1 & 0 & 1.3 \\
5.4 & 2 & 8.9 & 1.2 \\
5.7 & 3 & 1.8 & 1.3 \\
5.9 & 4 & 1.7 & 1.3 \\
6.3 & 5 & 2.1 & 1.2 \\
8.3 & 6 & 13.7 & 1.3 \\
8.9 & 7 & 5.2 & 1.2 \\
9.6 & 8 & 5.9 & 1.5 \\
\hline
\end{tabular}

two were $\mathrm{ACN} / \mathrm{H}_{2} \mathrm{O}$ in a ratio of $1: 1$ and $\mathrm{ACN} 99.9 \%$. All extraction experiments were performed using CBD hemp sample (A). The mass to volume ratio was chosen as proposed previously (Mudge et al. 2017). As shown in Fig. 3a, ACN 99.9\% yielded the lowest amount of extracted target analytes, especially for CBG, CBGA, THC, and THCA. However, it could be shown that $\mathrm{MeOH} /$ $\mathrm{H}_{2} \mathrm{O}$ (4:1) and $\mathrm{ACN} / \mathrm{H}_{2} \mathrm{O}$ (1:1) provided similar performance than the standard method. These two alternative solvents were even able to extract more CBGA, CBG, and CBD than $\mathrm{MeOH} / \mathrm{CHCl}_{3}$ (9:1). Finally, ACN/ $\mathrm{H}_{2} \mathrm{O}$ (1:1) was chosen because of better compatibility with starting conditions of the HPLC method. Furthermore, higher aqueous content renders this extraction system more environmentally friendly. According to UNODC, homogenized plant samples should be extracted with solvent in an ultrasonic bath for $15 \mathrm{~min}$ including several vortexing steps $(10 \mathrm{~s}$ each) (U.N.O.o. Drugs, Crime 2013). Several different extraction techniques were tested in duplicate. The performance of the four procedures was compared and shown as relative extracted yield of cannabinoids. Different extracting methods were plotted against the standard method (Fig. 3b). Samples extracted without vortexing yielded the same amount of cannabinoids as the standard method. Vortexing alone or shortening ultra-sonication time to 5 min lowered the yield to around 50\% for CBGA, THCA, CBGD, CBG, and THC and to about $70 \%$ for CBDA. These results clearly indicate the need for $15 \mathrm{~min}$ in the ultrasonic bath whereas the influence of vortexing was insignificant. Extraction efficiency (EE) was controlled by repeating extraction process three times on samples (F) and (P5) showing highest contents in CBD/CBDA and $\mathrm{THC} / \mathrm{THCA}$, respectively. In general, the $\mathrm{EE}$ shows the completeness of an extraction procedure of authentic samples. For CBD-rich cannabis samples with low amounts of THC/THCA, the EE for THC, CBDA, and CBD was greater than $94.4 \%$ after one extraction step. For all other analytes, an EE > 99\% was observed in the first step. For THC- and THCA-rich samples, first extraction yielded in an EE of around 99\% for THC/THCA whereas after the second extraction step, all target analytes were extracted completely (Fig. 4). Interestingly, in THC-/ THCA-rich samples, no CBD or CBDA was found. EE for THC and THCA was only around 90\% for the first extraction step. However, after the second extraction, no more target analytes were found in the samples.

\section{Method development and validation}

Since there is no cannabinoid-free cannabis matrix, extracts from a closely related plant were used as surrogate matrix. The use of hop (Humulus lupulus L.) appeared to be most appropriate, because it belongs to the same family (Cannabacea) as cannabis (Nuutinen 2018). Therefore, it 


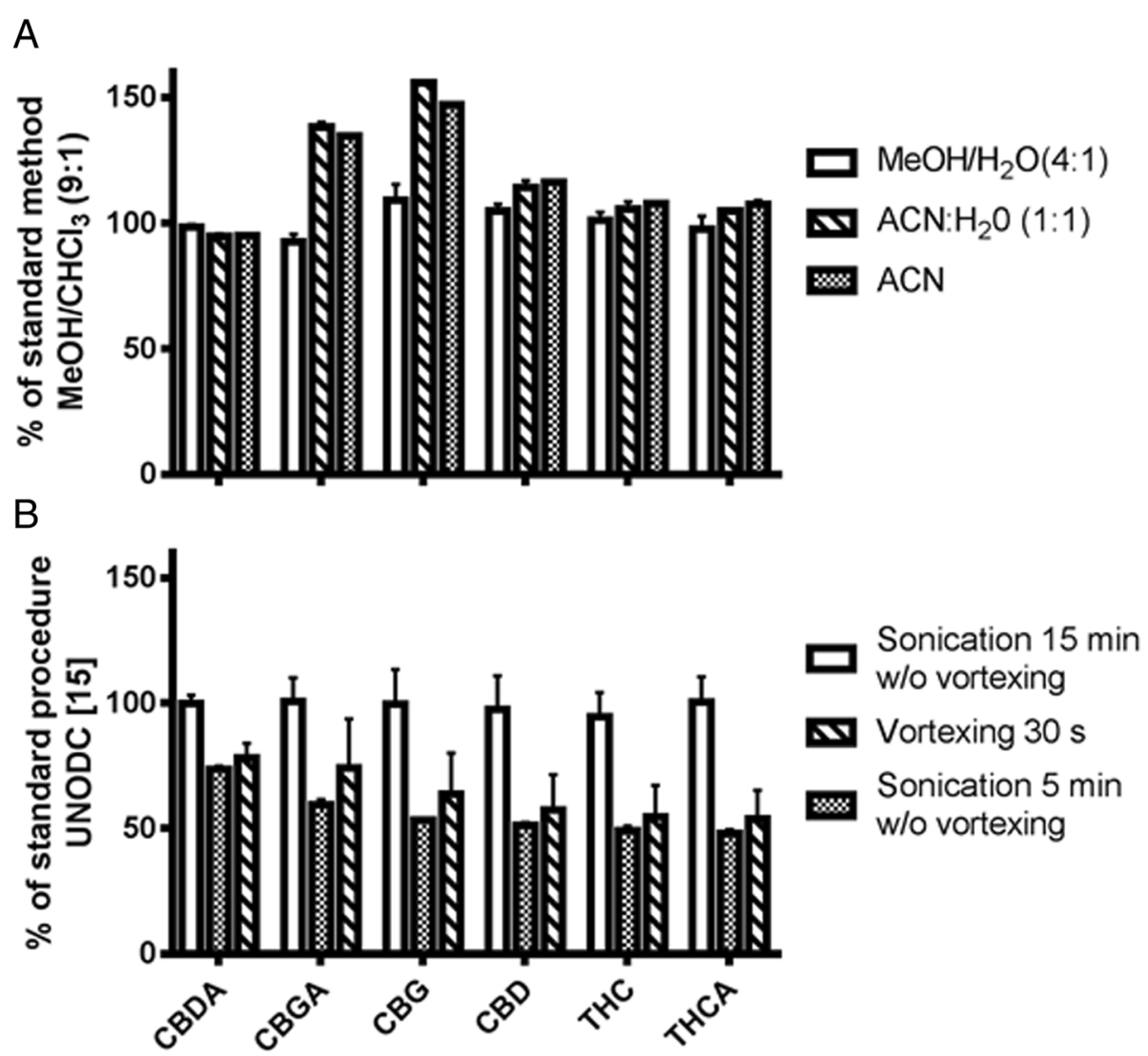

Fig. 3 a Data of different extraction solvents (a) and procedures (b)

is assumed that the general and non-specific composition and structure of hop is similar to cannabis. Blank extract of hop samples were investigated in detail, but no co-eluting substances in relation to any target analytes of cannabis were detected. The recovery effect (RE) of an analyte is the ratio of the detector response obtained from an amount of the analyte added before and after the extraction process to blank matrix or its extract, respectively. To calculate the RE, blank material was spiked with reference standards and re-extracted as described above $(N=$ 3 ). The extraction recovery effect was calculated using Eq.
1. Since there was a full recovery of all analytes observed (Table 2) and no co-eluting substance in the blank matrix was observed, a matrix-matched calibration was not necessary. As surrogate matrix for calibrants and quality control (QC) samples, the solvent mix $\mathrm{ACN} / \mathrm{H}_{2} \mathrm{O}(1: 1)$ is used. Four different concentrations of the analytes were chosen for the calibrations: $1,10,50$, and $100 \mu \mathrm{g} / \mathrm{mL}$. The dilutions were made using $\mathrm{ACN} / \mathrm{H}_{2} \mathrm{O}$ 1:1. For six following days, the analysis of four calibrants and two QC samples $\left(\mathrm{QC}_{\text {Low }}\right.$ and $\mathrm{QC}_{\text {High }}$ ) were performed. $\mathrm{QC}$ samples were analyzed in duplicate. Validation data is shown in

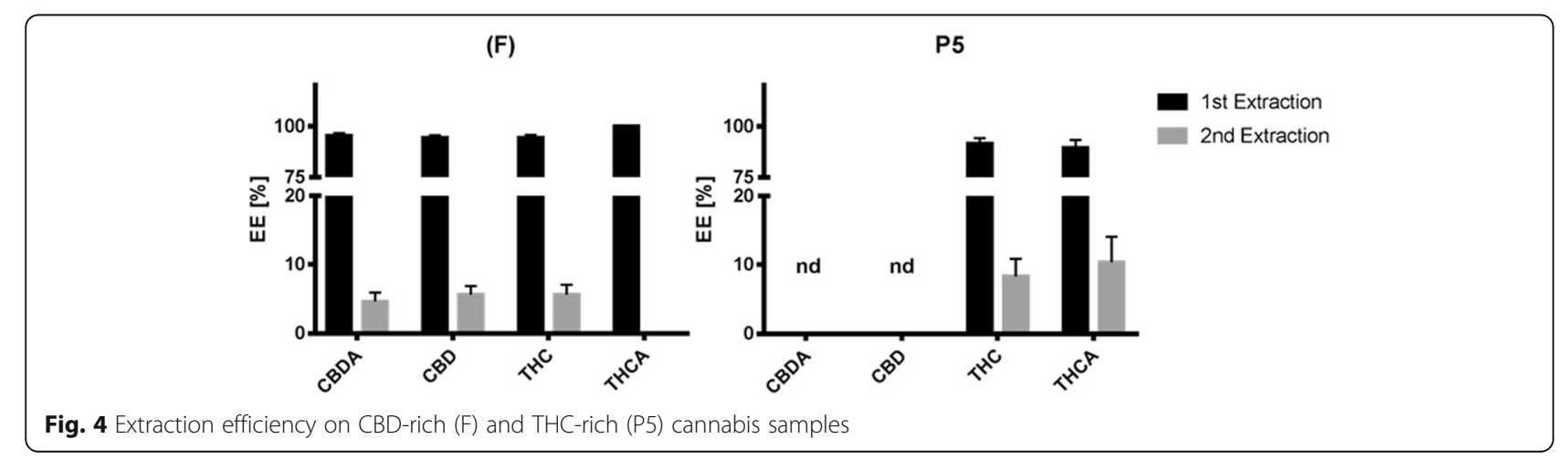


Table 2 Method validation data: recovery effect, bias, intra-day precision $\left(R_{S D}\right)$, interday-precision $\left(R_{S} D_{T}\right)$

\begin{tabular}{|c|c|c|c|c|c|c|c|c|c|}
\hline \multirow[t]{2}{*}{ Analyte } & \multirow[t]{2}{*}{ Cal. model } & \multirow[t]{2}{*}{$\mathrm{EE}(\%),(\mathrm{CV}, \%)$} & \multirow[t]{2}{*}{ RE (\%), (CV, \%) } & \multicolumn{3}{|l|}{ QC High } & \multicolumn{3}{|l|}{ QC Low } \\
\hline & & & & Bias (\%) & RSDT (\%) & RSDR (\%) & Bias (\%) & RSDT (\%) & RSDR (\%) \\
\hline CBDV & $1 / x^{2}$ & $>99.9$ & 103.1 & -0.2 & 4.7 & 5.6 & 2.9 & 4.6 & 11.9 \\
\hline CBDA & $1 / x^{2}$ & $95.3(1.2)$ & 105.9 & -2.3 & 2.6 & 6.1 & 3.1 & 4.3 & 11.3 \\
\hline CBGA & $1 / x^{2}$ & $>99.9$ & 104.3 & 0.4 & 3.3 & 4.4 & 1.4 & 9.4 & 16.3 \\
\hline CBG & $1 / x^{2}$ & $>99.9$ & 106.2 & 2.8 & 2.4 & 5.7 & -1.3 & 3.9 & 20.0 \\
\hline $\mathrm{CBD}$ & $1 / x^{2}$ & $94.4(1.3)$ & 106.8 & 3.9 & 2.1 & 5.7 & 5.2 & 4.3 & 9.9 \\
\hline CBN & $1 / x^{2}$ & $>99.9$ & 104.3 & -3.9 & 3.3 & 5.8 & 3.2 & 5.3 & 11.1 \\
\hline $\mathrm{THC}$ & Non-weighted & $94.4(1.5)$ & 102.7 & 8.6 & 1.1 & 1.9 & 10.3 & 6.2 & 12.7 \\
\hline THCA & $1 / x^{2}$ & $>99.9$ & 103.0 & -3.7 & 1.3 & 6.0 & 1.2 & 4.6 & 16.8 \\
\hline
\end{tabular}

Table 2. Bias for $\mathrm{QC}_{\mathrm{Low}}$ and $\mathrm{QC}_{\mathrm{High}}$ for all analytes varied between 1.3 and $-10.3 \%$ and 3.9 and $8.6 \%$, respectively. Blank samples were injected after the highest calibrant, and carry-over was not observed. Across the calibration range, $1 / x^{2}$ was found to be the best fit for all cannabinoids, whereas a non-weighted calibration was used for THC (Table 2). The decision on weighted calibration was made by comparing the deviations of the back-calculated concentrations from the respective nominal concentrations of the calibrators. Limit of quantification (LoQ) for all analytes was set to $1 \mu \mathrm{g} / \mathrm{mL}$ because of low bias and acceptable imprecision data. The limit of detection was not investigated systematically. Due to the early elution of the IS phenprocoumon, 60 different small molecules were screened for a second internal standard for late eluting peaks. Sixty different small molecules were screened for a second internal standard for use as a second internal standard for late eluting peaks. Under given chromatographic conditions, co-elution was observed with one of the target analytes. The list of selected compounds is given in the supporting information (Additional file 1: Table S3). Because of the acceptable bias and imprecision data, phenprocoumon was accepted as IS for all target analytes.

\section{Stability}

CBD-hemp sample (A) was analyzed after indicated time points. All analyses were performed in duplicate. In Fig. 5a, a time-dependent decline of the concentrations is observed for all three acidic cannabinoids (CBDA, CBGA, and THCA) and at the same time the formation of their decarboxylated forms (CBD, CBG, and THC) is detected. The decarboxylation process of THCA occurred more rapidly than for the other two acidic precursors. The concentrations of CBD, CBG, and THC show a saturation at different time points (Fig. 5a). Afterwards, the curves start to decline, indicating further conversion of these compounds. For CBD and CBG, the decline of the analyzed concentrations appeared to start roughly after $30 \mathrm{~h}$; however, for $\mathrm{THC}$, at about $4 \mathrm{~h}$. At the same time, an increase in $\mathrm{CBN}$ content could be observed. Starting from $0.14 \mu \mathrm{g} / \mathrm{mL}$ concentration of CBN increased to around $4.25 \mu \mathrm{g} / \mathrm{mL}$ after $72 \mathrm{~h}$. Therefore $\mathrm{CBN}$ concentration may be useful as quality marker for storage conditions of cannabis samples and should be further take into account for estimation of initial THC content.

For the UV stability experiment, sample (B) was placed in a weighing flask and exposed to UV light $(\lambda=350$ $750 \mathrm{~nm}$, Fig. 5b). Since the temperature under the UV lamp can slightly increase (up to $36^{\circ} \mathrm{C}$ ), a control, covered with aluminum foil, was also analyzed (Additional file 1: Figure S6A). The samples were quantified after indicated time points of exposure. Data in Fig. $5 \mathrm{~b}$ showed a time-dependent decrease of all target analytes under UV-light exposure. A fivefold increase in CBN content could be detected, from approximately 0.2 to $1 \mu \mathrm{g} / \mathrm{mL}$, after $8 \mathrm{~h}$. Interestingly, CBGA and CBG were more susceptible to UV light compared to heat conditions. In the control, only a slight increase in $\mathrm{CBN}$ and decrease of THCA was observed.

\section{Quantification}

THC and THCA concentrations of the 12 forensic hemp samples were analyzed using the developed HPLC-UV method. Results were compared with results obtained by the forensic institute Zurich using standard GC-FID method. In general, correlation of both methods was high $\left(R^{2}=0.956\right.$, Fig. 6). For all samples, the difference between the expected and the found THC+THCA content was between -0.3 and $2.4 \%$ (Additional file 1: Table S4). The slope of the regression line and $y$-intercept in both cases was 1.033 and 0.447 , respectively. In general, THC/THCA were quantified higher by the HPLC-based method than the GC method. This could be explained by incomplete conversion of THCA to THC or conversion of THC to $\mathrm{CBN}$ at higher temperatures. Dussy et al. showed that at $150{ }^{\circ} \mathrm{C} \mathrm{THC}$ reaches its stability optimum for an accurate analysis, and above that temperature, the conversion to $\mathrm{CBN}$ takes place (Dussy et al. 2005). Interestingly, in forensic samples, only 

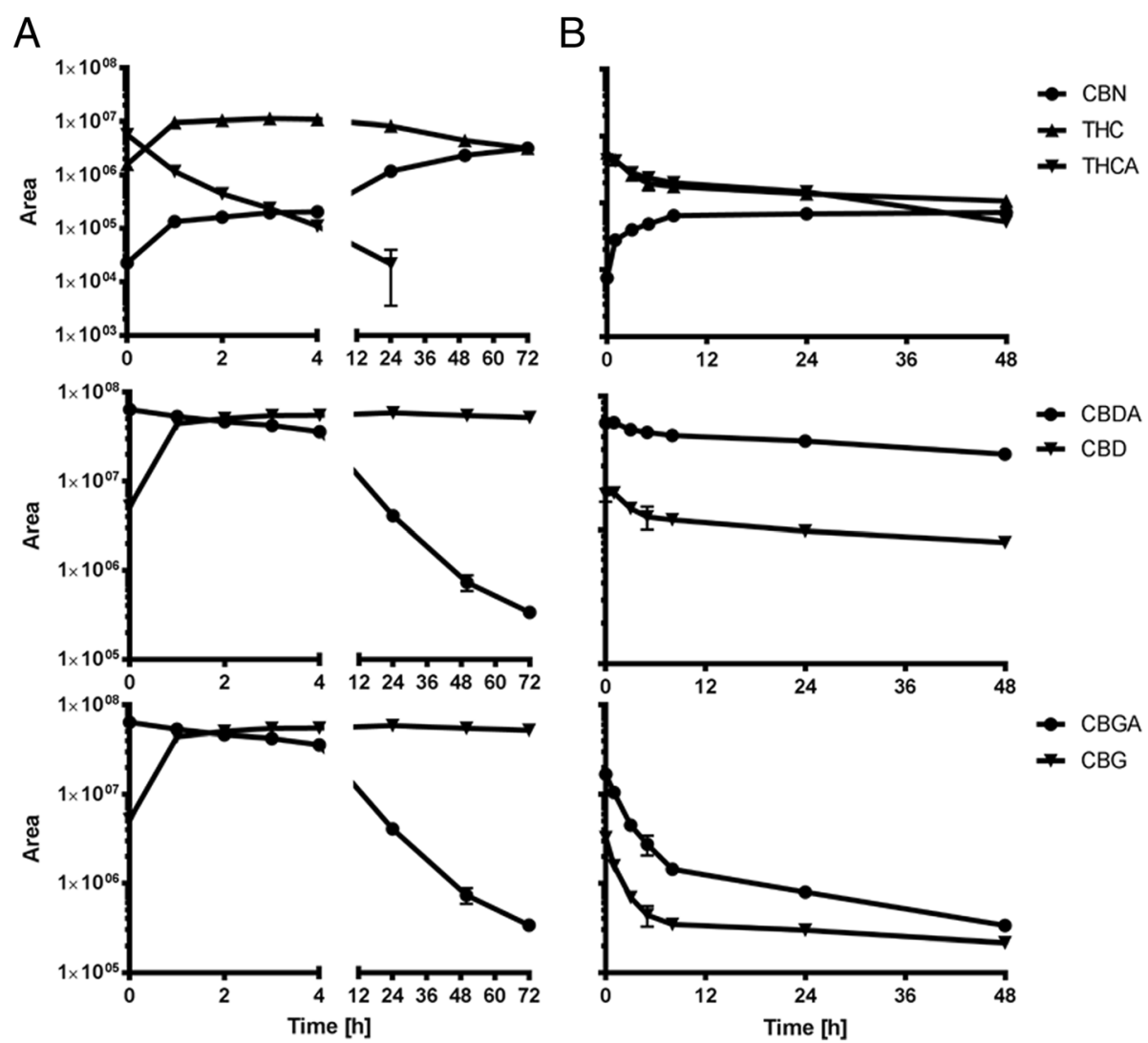

Fig. 5 Heat stability (a) and UV-stability (b) studies on seven cannabinoids

THC/THCA and CBD/CBDA were detected. Other target analytes were found only in traces. The cannabinoid content of eight different legally sold CBD-hemp tobacco samples obtained in Switzerland were analyzed. For only two out of eight samples (B, C), a certificate of analysis was available online. As indicated in the

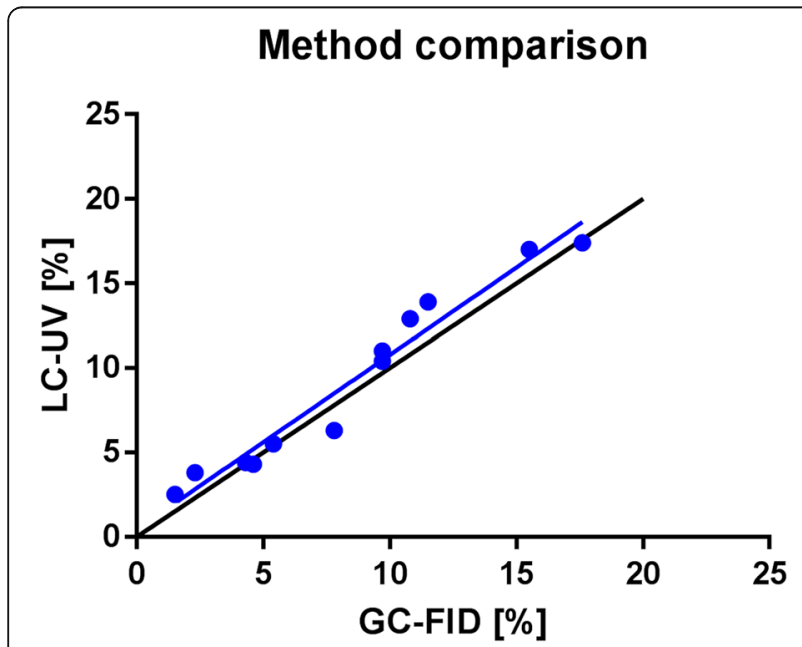

Fig. 6 Method comparison of overall THC/THCA content determination by GC-FID and LC-UV certificate, reported cannabinoid values were not batch specific. For nearly all samples, CBD content was far below the declared values. Only for sample (C), CBD content was higher than indicated. In general, it can be observed that the THC+THCA contents of all tobacco samples were under the maximal legally allowed limit of $1 \%$. CBDV and CBN were below the detection limit. Nevertheless, our analysis also showed that samples (F) and $(\mathbf{H})$ with a concentration of $0.91 \%$ and $0.90 \%$ respectively were very close to this threshold. Since CBD tobacco is sold in whole plant pieces, the content of the cannabinoids can vary depending on which part of the flower is ripped and ground and used for analysis, because different parts of the plant produce different amount of substances. To minimize such fluctuations, all batches should be homogenized before being analyzed and sold.

\section{Loss on drying}

The content of volatile compounds in crude drugs is an important factor since it influences the concentrations of the substances with pharmacological activity. Through manipulation, a lower content of target compounds could be reported. The current monograph on cannabis presented in the German Pharmacopoeia does not 
Table 3 Content of cannabinoids in commercial available hemp samples. Declared values were given in parenthesis. Values written in italics are below the LoQ

\begin{tabular}{lllllllll}
\hline [\%] & $(\mathrm{A})$ & $(\mathrm{B})$ & $(\mathrm{C})$ & $(\mathrm{D})$ & $(\mathrm{E})$ & $(\mathrm{F})$ & $(\mathrm{G})$ & $(\mathrm{H})$ \\
\hline CBDA & 4.5 & 12.5 & $16.1(13.7)$ & $12.6(14)$ & 5.8 & 13.3 & 13.2 & 17.3 \\
CBD & 0.3 & 1.6 & $1.0(0.4)$ & $0.5(0.4)$ & 9.8 & 6.5 & 1.6 & 2.5 \\
CBD+CBDA & $4.8(18)$ & - & - & - & $15.6(21)$ & - & $14.8(18)$ & $19.8(23)$ \\
THC & 0.1 & 0.2 & $0.1(0.05)$ & $0.05(0.05)$ & 0.6 & 0.7 & 0.2 & 0.3 \\
THCA & 0.4 & 0.4 & $0.6(0.6)$ & $0.5(0.6)$ & 0 & 0.2 & 0.5 & 0.6 \\
THC+THCA & $0.5(<1)$ & $0.6(<1)$ & - & - & $0.6(<1)$ & $0.9(<1)$ & $0.7(0.8)$ & $0.9(0.9)$ \\
CBGA & $<\mathrm{nd}$ & 1.49 & 0.90 & 0.56 & 0.24 & 0.14 & 0.3 & 0.15 \\
CBG & $<\mathrm{nd}$ & 0.24 & 0.17 & 0.24 & $<\mathrm{nd}$ & 0.15 & 0.06 & 0.12 \\
CBDV & $<\mathrm{nd}$ & $<\mathrm{nd}$ & $<\mathrm{nd}$ & $<\mathrm{nd}$ & $<\mathrm{nd}$ & $<\mathrm{nd}$ & $<\mathrm{nd}$ & $<\mathrm{nd}$ \\
CBN [\%] & $<\mathrm{nd}$ & $<\mathrm{nd}$ & $<\mathrm{n} . \mathrm{d}$ & $<\mathrm{nd}$ & $<\mathrm{nd}$ & $<\mathrm{nd}$ & $<\mathrm{nd}$ & $<\mathrm{nd}$ \\
Loss on drying & 5.8 & 4.3 & 6.5 & 4.4 & 8.3 & 7.7 & 8.8 & 5.9 \\
\hline
\end{tabular}

nd not detected

provide any information about loss on drying (BfArM 2017). The monograph of hop includes drying the sample at $105^{\circ} \mathrm{C}$ for $2 \mathrm{~h}$. The loss on drying of hop was performed as described in the Ph. Eur. and additionally till constant mass was achieved after drying at $60^{\circ} \mathrm{C}$. Both procedures resulted in loss of $3.8 \%$ (Table 3 ). Since the cannabinoids are heat instable, hemp samples were dried till constant mass at $60^{\circ} \mathrm{C}$. The loss on drying of all tested CBD-hemp samples is shown in Table 3. The highest loss was determined in samples $(\mathbf{E})$ and $(\mathbf{G})$ and was higher than $8 \%$. The lowest loss of $4.5 \%$ was determined in sample (B). In general, all hemp samples showed a higher loss on drying compared to hop samples.

\section{Conclusion}

We developed a fast and reproducible HPLC-UV method for the quantification of hemp samples. Method validation confirmed that the method produces repeatable and accurate results for eight different cannabinoids in less time. The use of a water to acetonitrile mixture (50\%) for extraction instead of chlorinated organic solvent mixtures renders this method more ecologically friendly The presented procedure is universally applicable in a wide range of settings from pharmacopeial monographs, research, quality control, and regulatory evaluation of this emerging field of herbal industry. Additionally, the transfer to an UHPLC-DAD system reduced the analysis time to less than 5 min providing additional ecological benefits. An alternative loss on drying experiment was further described and showed similar results when applied on closely related hop samples. CBN content could be used as a marker for storage control of cannabis samples. The results obtained from the analysis of authentic samples highlight the need for accurate determination of the cannabinoids concentrations in regularly time intervals of different $C$. sativa L. strains to limit the risk of increased THC content in legalized products and to provide characterized products for therapeutic use.

\section{Additional file}

Additional file 1: Table S1. Use d RP-HPLC columns. Table S2. Gradient elution HPLC method. Table S3. Used compounds for use as internal standard. Table S4. Results obtained by GC- and LC-based methods. Figure S5. UHPLC-UV chromatogram of Cal 2 recorded at $\lambda=220 \mathrm{~nm}$. Figure S6. UV experiment with covered samples (A) and non-covered samples (B). (DOCX $246 \mathrm{~kb})$

\section{Abbreviations}

ACN: Acetonitrile; C. sativa: Cannabis sativa; Cal: Calibrator; CBD: Cannabidiol; CBDA: Cannabidiolic acid; CBDV: Cannabidivarin; CBG: Cannabigerol; CBGA: Cannabigerolic acid; CBN: Cannabinol; $\mathrm{CHCl}_{3}$ : Chloroform; EE: Extraction efficiency; EtOH: Ethanol; FA: Formic acid; FID: Flame ionization detector; GC: Gas chromatography; HPLC: High-performance liquid chromatography; IS: Internal standard; LC: Liquid chromatography; LoD: Limit of detection; LoQ: Limit of quantification; ME: Matrix effect; $\mathrm{MeOH}$ : Methanol; Ph. Eur.: Pharmacopoea europaea; QC: Quality control; RE: Recovery effect; RP: Reversed phase; RSD: Relative standard deviation; THC: $\Delta 9$ Tetrahydrocannabinol; THCA: $\triangle$ 9-Tetrahydrocannabinolic acid; UHPLC: Ultra-high-performance liquid chromatography; UV: Ultraviolet

\section{Acknowledgements}

The authors want to acknowledge Danielle Luethi for excellent support in laboratory handling of all samples. We further thank the Forensic Institute of Zurich for providing and analyzing confiscated hemp samples.

\section{Funding}

This work has not been financially supported.

\section{Availability of data and materials}

Research data have been provided in the manuscript and supporting information.

\section{Authors' contributions}

This study was designed by CS. SZ and RA equally performed the experimental work. The manuscript was written through the contributions of all authors. All authors have given approval to the final version of the manuscript.

Competing interests

The authors declare that they have no competing interests. 


\section{Publisher's Note}

Springer Nature remains neutral with regard to jurisdictional claims in published maps and institutional affiliations.

Received: 11 October 2018 Accepted: 7 November 2018

Published online: 24 November 2018

\section{References}

Amada N, Yamasaki Y, Williams CM, Whalley BJ. Cannabidivarin (CBDV) suppresses pentylenetetrazole (PTZ)-induced increases in epilepsy-related gene expression. PeerJ. 2013;1:e214.

Ambach L, Penitschka F, Broillet A, Konig S, Weinmann W, Bernhard W. Simultaneous quantification of delta-9-THC, THC-acid A, CBN and CBD in seized drugs using HPLC-DAD. Forensic Sci Int. 2014;243:107-11.

BfArM, Cannabisblüten - Cannabis flos 2017, https://www.bfarm.de/SharedDocs/ Downloads/DE/Arzneimittel/Zulassung/ZulRelThemen/azbuch/Monografie_ Cannabisblueten.pdf;jsessionid=8F09720B7BADA085700EE676CF2DA645.2_ cid354?_blob=publicationFile\&v=2,

Brighenti V, Pellati F, Steinbach M, Maran D, Benvenuti S. Development of a new extraction technique and HPLC method for the analysis of non-psychoactive cannabinoids in fibre-type Cannabis sativa L. (hemp). J Pharm Biomed Anal. 2017:143:228-36.

Calvi L, Pentimalli D, Panseri S, Giupponi L, Gelmini F, Beretta G, Vitali D, Bruno M, Zilio E, Pavlovic R, Giorgi A. Comprehensive quality evaluation of medical Cannabis sativa L. inflorescence and macerated oils based on HS-SPME coupled to GC-MS and LC-HRMS (q-exactive orbitrap(R)) approach. J Pharm Biomed Anal. 2018;150:208-19.

Citti C, Braghiroli D, Vandelli MA, Cannazza G. Pharmaceutical and biomedical analysis of cannabinoids: a critical review. J Pharm Biomed Anal. 2018b;147:565-79.

Citti C, Pacchetti B, Vandelli MA, Forni F, Cannazza G. Analysis of cannabinoids in commercial hemp seed oil and decarboxylation kinetics studies of cannabidiolic acid (CBDA). J Pharm Biomed Anal. 2018a;149:532-40.

Corroon J, Phillips JA. A cross-sectional study of cannabidiol users. Cannabis Cannabinoid Res. 2018;3(1):152-61.

De Backer B, Debrus B, Lebrun P, Theunis L, Dubois N, Decock L, Verstraete A, Hubert P, Charlier C. Innovative development and validation of an HPLC/ DAD method for the qualitative and quantitative determination of major cannabinoids in cannabis plant material. J Chromatogr B Analyt Technol Biomed Life Sci. 2009;877(32):4115-24.

Dussy FE, Hamberg C, Luginbuhl M, Schwerzmann T, Briellmann TA. Isolation of Delta9-THCA-A from hemp and analytical aspects concerning the determination of Delta9-THC in cannabis products. Forensic Sci Int. 2005; 149(1):3-10.

EISohly MA, Salem M. Chapter 5 cannabinoids analysis: analytical methods for different biological specimens. In: Bogusz MJ, editor. Handbook of analytical separations, Elsevier science B.V; 2000. p. 163-93.

Machado Rocha FC, Stefano SC, De Cassia Haiek R, Rosa Oliveira LM, Da Silveira DX. Therapeutic use of Cannabis sativa on chemotherapy-induced nausea and vomiting among cancer patients: systematic review and meta-analysis. Eur J Cancer Care (Engl). 2008;17(5):431-43.

Mudge EM, Murch SJ, Brown PN. Leaner and greener analysis of cannabinoids. Anal Bioanal Chem. 2017:409(12):3153-63.

Nuutinen T. Medicinal properties of terpenes found in Cannabis sativa and Humulus lupulus. Eur J Med Chem. 2018;157:198-228.

Patel B, Wene D, Fan ZT. Qualitative and quantitative measurement of cannabinoids in cannabis using modified HPLC/DAD method. J Pharm Biomed Anal. 2017;146:15-23.

Pavlovic R, Nenna G, Calvi L, Panseri S, Borgonovo G, Giupponi L, Cannazza G, Giorgi A. Quality traits of "cannabidiol oils": cannabinoids content, terpene fingerprint and oxidation stability of European commercially available preparations. Molecules. 2018;23(5):1230-51.

T. Pellechia, Legal cannabis industry poised for big growth, in north america and around the world. $2018 \mathrm{https} / /$ www.forbes.com/sites/thomaspellechia/2018/ 03/01/double-digit-billions-puts-north-america-in-the-worldwide-cannabismarket-lead/\#53ea3ac26510, Forbes, (accessed Septemper 22.2018).

F.T. Peters, M. Hartung, M. Herbold, G. Schmitt, T. Daldrup, F. Mußhoff, APPENDIX B Requirements for the validation of analytical methods, Toxichem Krimtech 76 (2009) 185-208.

Ph. Eur., Loss on drying(2.2.32), 2018; 9.6: 72-73.

S. Sirikantaramas, F. Taura, Cannabinoids: biosynthesis and biotechnological applications, in: S. Chandra, H. Lata, M.A. ElSohly (Eds.), Cannabis sativa L. - botany and biotechnology, Springer International Publishing, Cham, 2017, pp. 183-206.

Thomas A, Baillie GL, Phillips AM, Razdan RK, Ross RA, Pertwee RG. Cannabidiol displays unexpectedly high potency as an antagonist of $\mathrm{CB} 1$ and $\mathrm{CB} 2$ receptor agonists in vitro. Br J Pharmacol. 2007;150(5):613-23.

U.N.O.o. Drugs, Crime, Recommended methods for the identification and analysis of Cannabis and Cannabis products, 2013.

Velasco G, Sanchez C, Guzman M. Anticancer mechanisms of cannabinoids. Curr Oncol. 2016;23(2):S23-32

Wang M, Wang YH, Avula B, Radwan MM, Wanas AS, Mehmedic Z, van Antwerp J, ElSohly MA, Khan IA. Quantitative determination of cannabinoids in Cannabis and Cannabis products using ultra-high-performance supercritical fluid chromatography and diode array/mass spectrometric detection. J Forensic Sci. 2017;62(3):602-11.

Wang M, Wang Y-H, Avula B, Radwan MM, Wanas AS, van Antwerp J, Parcher JF, ElSohly MA, Khan IA. Decarboxylation study of acidic cannabinoids: a novel approach using ultra-high-performance supercritical fluid chromatography/ photodiode array-mass spectrometry. Cannabis and Cannabinoid Research. 2016;1 (1):262-71.

Zoller O, Rhyn P, Zimmerli B. High-performance liquid chromatographic determination of delta9-tetrahydrocannabinol and the corresponding acid in hemp containing foods with special regard to the fluorescence properties of delta9-tetrahydrocannabinol. J Chromatogr A. 2000;872(1-2):101-10.

\section{Submit your manuscript to a SpringerOpen ${ }^{\circ}$ journal and benefit from:}

- Convenient online submission

- Rigorous peer review

- Open access: articles freely available online

High visibility within the field

- Retaining the copyright to your article

Submit your next manuscript at $\boldsymbol{\nabla}$ springeropen.com 